\title{
Actualités sur le Covid-19
}

\author{
P.-G. Claret $\cdot$ J. Treille $\cdot$ P. Loubet $\cdot$ C. Freitag $\cdot$ J.-P. Beregi $\cdot$ J.E. de La Coussaye
}

C SFMU et Lavoisier SAS 2020

\section{Clinical characteristics of 50466 hospitalized patients with 2019-nCoV infection}

Sun P, Qie S, Liu Z, et al (2020) J Med Virol [in press]

Méta-analyse chinoise incluant dix études [1-6] portant au total sur 50466 patients présentant une infection à Covid-19. Les auteurs montrent que les incidences de l'hyperthermie, de la toux et des myalgies sont, respectivement, de 89, 72 et $42 \%$. Une tomodensitométrie anormale est retrouvée dans $97 \%$ des cas. La survenue d'un syndrome de détresse respiratoire aigu est retrouvée dans $15 \%$ des cas et la mortalité est de 4,3\%.

\section{P.-G. Claret}

Faculté de médecine Montpellier-Nîmes, université de Montpellier, F-34060 Montpellier, France

Pôle Anesthésie-Réanimation-Douleur-Urgences,

CHU de Nîmes, F-30000 Nîmes, France

J. Treille

Pôle Anesthésie-Réanimation-Douleur-Urgences,

CHU de Nîmes, F-30000 Nîmes, France

P. Loubet

Service de maladie infectieuse, CHU de Nîmes,

F-30000 Nîmes, France

C. Freitag

Service d'imagerie, CHU de Nîmes, F-30000 Nîmes, France

\section{J.-P. Beregi}

Faculté de médecine Montpellier-Nîmes, université de Montpellier, F-34060 Montpellier, France

Service d'imagerie, CHU de Nîmes, F-30000 Nîmes, France

J.E. de La Coussaye

Faculté de médecine Montpellier-Nîmes, université de Montpellier, F-34060 Montpellier, France Pôle Anesthésie-Réanimation-Douleur-Urgences,

CHU de Nîmes, F-30000 Nîmes, France
The incubation period of coronavirus disease 2019 (Covid-19) from publicly reported confirmed cases: estimation and application

Lauer SA, Grantz KH, Bi Q, et al (2020) Ann Intern Med [in press]

Étude américaine multicentrique, observationnelle, incluant via les données accessibles par les médias 181 patients présentant une infection à Covid-19 en Chine. Les auteurs montrent que la médiane d'incubation est de $5,1 \mathrm{j}$ (intervalle de confiance à $95 \%:[4,5-5,8])$ et que $97 \%$ des patients développent des symptômes $11 \mathrm{j}$ [8-16] après l'infection. Ces estimations impliquent que, selon des hypothèses prudentes, $1 \%$ des patients développeront des symptômes après les $14 \mathrm{j}$ de quarantaine.

Early epidemiological analysis of the coronavirus disease 2019 outbreak based on crowdsourced data: a population level observational study

Sun K, Chen J, Viboud (2020) Lancet Digital Health Étude chinoise multicentrique, observationnelle, incluant 507 patients présentant des infections à Covid-19 rapportées au travers de réseaux sociaux en Chine (364) et hors de Chine (143). Malgré seulement 507 patients inclus, les caractéristiques de la population étudiées sont similaires à celles d'études plus importantes sur le sujet. Les auteurs observent que peu de patients infectés ont moins de 15 ans (3\%). Le profil d'âge des patients inclus, ajusté aux données démographiques de base pour chaque pays, confirme un pourcentage d'infection moins important chez les enfants.

Clinical course and outcomes of critically ill patients with SARS-CoV-2 pneumonia in Wuhan, China: a single-centered, retrospective, observational study

Yang X, Yu Y, Xu J, et al (2020) Lancet Respir Med [in press] 
Étude chinoise monocentrique, observationnelle, incluant 52 patients présentant une infection à Covid-19 et admis en réanimation. L'âge moyen des patients était de 60 ans (écart-type $=13$ ), $67 \%$ étaient des hommes $(\mathrm{n}=35)$, et $40 \%$ présentaient des comorbidités chroniques (21). L'hyperthermie était observée chez $98 \%$ des patients $(\mathrm{n}=51)$. Le pourcentage de mortalité à $28 \mathrm{j}$ était de $61 \%(\mathrm{n}=32)$.

\section{Clinical course and risk factors for mortality of adult inpatients with Covid-19 in Wuhan, China}

Zhou F, Yu T, Du R, et al (2020) Lancet [in press]

Étude chinoise bicentrique, observationnelle, incluant sur le mois de janvier 2020 l'ensemble des 191 patients présentant une infection à Covid-19 et nécessitant une hospitalisation. L'âge médian des patients était de 56 ans (écart interquartile: [46-67]), et $62 \%$ étaient des hommes $(\mathrm{n}=119)$. Cinquante-quatre $(28 \%)$ patients sont décédés à l'hôpital. En analyse multivariée, le décès était associé à l'âge (odds ratio $[O R]=1,10$; intervalle de confiance à 95\%: [1,03-1,17]), à un score SOFA (Sepsis-related Organ Failure Assessment) élevé (OR = 5,65 [2,6112,23]) et à un taux de d-dimères supérieur à 1 microg/l (18,42 [2,64-128,55]). La durée médiane de l'excrétion virale était de $20 j$ [17-24] chez les survivants, et le virus était détectable jusqu'au décès chez les non-survivants.

\section{Clinical characteristics of coronavirus disease 2019 in China}

Guan W, Ni Z, Hu Y, et al (2020) N Engl J Med [in press]

Étude chinoise multicentrique, observationnelle, incluant 1099 patients présentant une infection à Covid-19. L'âge médian des patients était de 47 ans (écart interquartile: [35-58]), et $58 \%$ étaient des hommes $(\mathrm{n}=640)$. L'hyperthermie n'était présente à l'admission que chez $44 \%$ des patients, puis chez $89 \%$ des patients au cours de l'hospitalisation. Soixante-sept patients (6,1\%) présentaient un

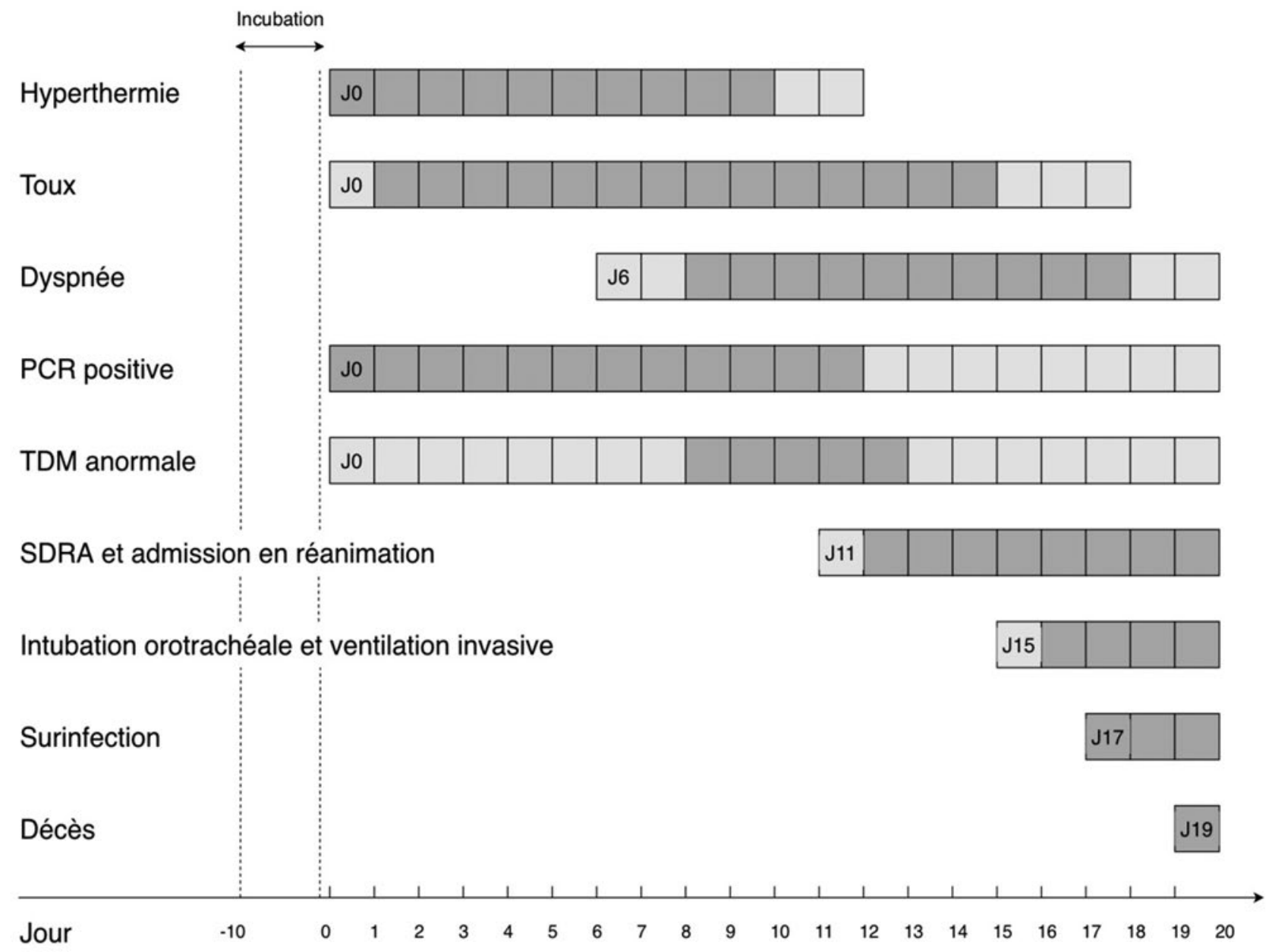

Fig. 1 Évolutions clinique, paraclinique et de la prise en charge des patients Covid-19 en cas de complication [7-10] (PCR : réaction en chaîne par polymérase ; SDRA : syndrome de détresse respiratoire aiguë ; TDM : tomodensitométrie) 
événement indésirable grave: admission en réanimation $(5,0 \%)$, ventilation invasive $(2,3 \%)$ ou décès $(1,4 \%)$ durant l'hospitalisation. Seulement 2,9\% des tomodensitométries thoraciques ou radiographies du thorax réalisées chez les patients graves étaient normales.

Time course of lung changes on chest $\mathrm{CT}$ during recovery from 2019 novel coronavirus (Covid-19) pneumonia

Pan F, Ye T, Sun P, et al (2020) Radiology [in press]

Étude chinoise monocentrique, observationnelle, incluant 21 patients hospitalisés pour une infection à Covid-19, bénéficiant d'une tomodensitométrie (TDM) pulmonaire puis rentrant au domicile. Les auteurs montrent que le maximum des lésions pulmonaires est observé au $10^{\circ}$ jour d'hospitalisation. Quatre phases d'évolution radiologique du parenchyme pulmonaire sont décrites: 1) de 0 à $4 j$, des opacités en verre dépoli; 2) de 5 à $8 j$, un aspect en mosaïques (crazy paving); 3 ) de 3 à $13 j$, une condensation importante et un maximum des lésions ; 4) plus de $14 j$, une diminution des lésions observées.

\section{Correlation of chest CT and RT-PCR testing in coronavirus disease 2019 (Covid-19) in China: a report of 1,014 cases}

Ai T, Yang Z, Hou H, et al (2020) Radiology [in press]

Étude chinoise monocentrique, observationnelle, incluant 1014 patients présentant une suspicion clinique d'infection à Covid-19 et bénéficiant d'une tomodensitométrie (TDM) pulmonaire associée à une réaction en chaîne par polymérase (PCR). L'âge moyen des patients était de 51 ans (écart-type $=15$ ). En utilisant comme méthode de référence la PCR, les caractéristiques de la TDM pour le diagnostic de Covid-19 étaient : sensibilité $=97 \%$ (intervalle de confiance à $95 \%$ : [95-98]); spécificité $=25 \%$ [22-30] ; valeur prédictive négative $=83 \%$ [76-89]; valeur prédictive positive $=65 \%[62-68]$.

\section{Features, evaluation and treatment coronavirus (Covid-19)}

Cascella M, Rajnik M, Cuomo A, et al (2020) StatPearls Publishing [in press]

Article didactique italien synthétisant les données de la littérature récente, de la physiopathologie jusqu'aux possibles thérapeutiques. Parmi ces dernières, les auteurs rappellent qu'il n'existe pas, à ce jour, de traitement spécifique du Covid-19. Ils rappellent l'utilisation souvent nécessaire de l'oxygénothérapie nasale à haut débit, de la ventilation non invasive et de l'intubation orotrachéale pour ventilation mécanique. Ils précisent certaines thérapeutiques rarement utilisées (interféron alpha, lopinavir, ritonavir, remdesivir).

\section{Care for critically III patients with Covid19}

Murthy S, Gomersall CD, Fowler RA, et al (2020) JAMA [in press]

Article de synthèse concernant la prise en charge des patients critiques suite à une infection à Covid-19. Les auteurs rappellent que pour ces patients la durée médiane entre l'admission à l'hôpital et celle en réanimation est d'une dizaine de jours. En absence de traitement spécifique, la prise en charge est similaire aux autres défaillances respiratoires sur pneumopathie virale (ventilation protectrice, préserver l'hémodynamique, antibiothérapie si surinfection,
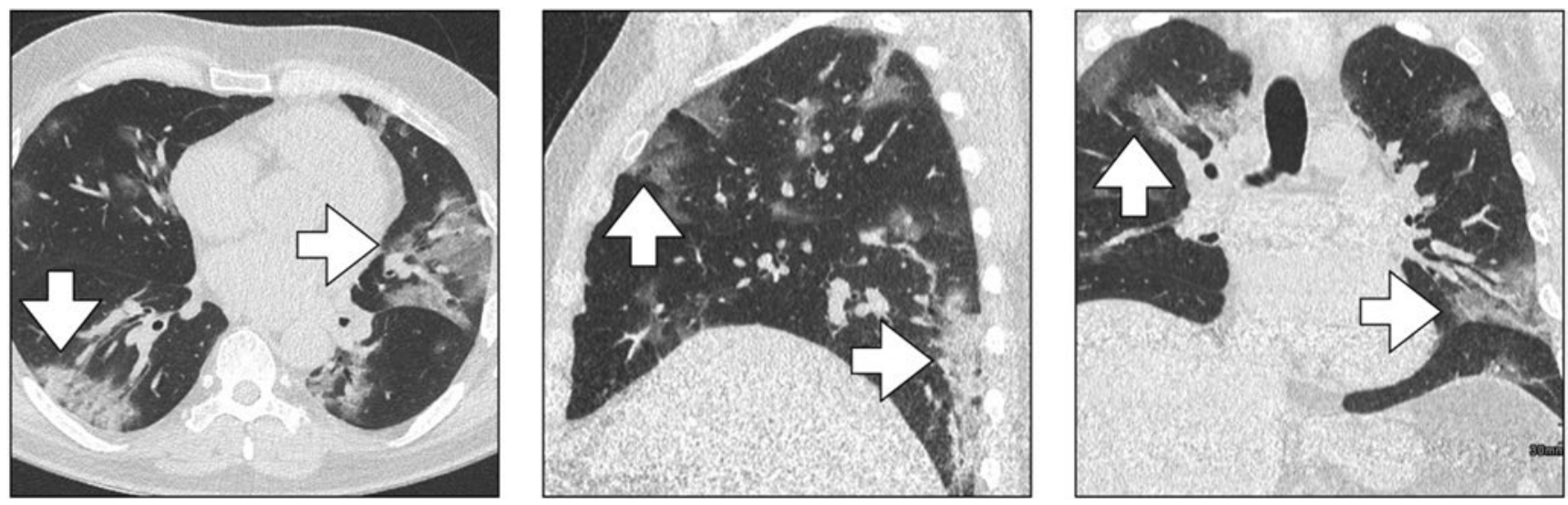

Fig. 2 Tomodensitométrie de patient présentant une infection à Covid-19. Présence de plusieurs zones en verre dépoli, bilatérales et en périphérie, avec image de mosaïque (crazy paving) 
décubitus ventrale, discuter l'oxygénation par membrane extracorporelle (ECMO, extracorporeal membrane oxygenation). Les auteurs soulignent que la ventilation non invasive et l'oxygénothérapie nasale à haut débit peuvent être des solutions d'attente si elles sont réalisées correctement (risque d'aérosolisation).

\section{Clinical predictors of mortality due to Covid-19 based on an analysis of data of 150 patients from Wuhan, China}

Ruan Q, Yang K, Wang W, et al (2020) Intensive Care Med [in press]

Étude chinoise bicentrique, observationnelle, incluant 150 patients présentant une infection à Covid-19 et admis en hospitalisation. Au total, la mortalité intrahospitalière était de $45 \%(\mathrm{n}=68)$. Les variables associées à un décès intrahospitalier étaient l'âge élevé, la présence de comorbidité, d'une surinfection bactérienne et l'élévation des marqueurs inflammatoires (CRP, ferritine, interleukine-6). Les causes de décès étaient une défaillance respiratoire chez $53 \%$ des patients, une défaillance cardiaque sur myocardite aiguë chez $7 \%$ des patients et une association des deux dans $33 \%$ des cas.

\section{Acute Physiology and Chronic Health Evaluation II score as a predictor of hospital mortality in patients of coronavirus disease 2019}

Zou X, Li S, Fang M, et al (2020) Crit Care Med [in press]

Étude chinoise monocentrique, observationnelle, incluant 154 adultes présentant une infection sévère à Covid-19 (mortalité intrahospitalière $=33 \%$ ). De tous les scores étudiés, le score APACHE II (Acute Physiology and Chronic Health Evaluation II) était le meilleur pour pronostiquer la mortalité des patients avec une aire sous la courbe de 0,966 (intervalle de confiance : [0,942-0,990]). Avec la meilleure valeur discriminante (17) du score, la sensibilité était de $96 \%$ et la spécificité de $86 \%$.

\section{Development and validation of a clinical risk score to predict the occurrence of critical illness in hospitalized patients with Covid-19}

Liang W, Liang H, Ou L, et al (2020) JAMA Intern Med [in press]

Étude chinoise multicentrique, observationnelle, incluant 2300 adultes (1 590 pour la cohorte de développement et 710 pour celle de validation) hospitalisés pour une infection à Covid-19 (mortalité intrahospitalière $=3,2 \%$ ).
Les auteurs observent les caractéristiques des patients entre le groupe de ceux présentant un événement indésirable grave (EIG; admission en réanimation, ventilation non invasive) et les autres. Dix variables indépendantes étaient prédictives d'un EIG : anomalie à l'imagerie thoracique (odds ratio $=3,39$; intervalles de confiance à $95 \%:[2,14-5,38])$, âge $(1,03$ [1,01-1,05]), hémoptysie (4,53 [1,36-15,15]), dyspnée (1,88 [1,18-3,01]), perte de conscience $(4,71$ [1,39-15,98]), nombre de comorbidités (1,60 [1,27-2,00]), antécédents de cancer (4,07 [1,2313,43]), rapport neutrophiles/lymphocytes (1,06 [1,021,10]), lactate déshydrogénase (1,002 [1,001-1,004]), bilirubine $(1,15$ [1,06-1,24]). L'aire sous la courbe était de 0,88 pour les deux cohortes.

\section{Characteristics and clinical significance of myocardial injury in patients with severe coronavirus disease 2019}

Shi S, Qin M, Cai Y, et al (2020) Eur Heart J [in press]

Étude chinoise monocentrique, observationnelle, incluant 671 adultes présentant une infection à Covid-19 (mortalité intrahospitalière $=9,2 \%$ ). Les auteurs observent les caractéristiques des patients entre le groupe de ceux présentant un infarctus du myocarde $(\mathrm{n}=106)$ et les autres. En analyse multivariée, l'atteinte myocardique chez ses patients Covid-19 était associée à l'âge lodds ratio $[O R]=1,64$ par période de dix ans; intervalle de confiance : $[1,28-2,10])$, aux antécédents d'hypertension $(O R=3,30[1,77-6,14])$, coronarien $(O R=2,92[1,32-$ $6,48])$, d'insuffisance rénale $(O R=9,03[2,43-33,59])$, de bronchopneumopathie chronique $(O R=4,01 \quad[1,28$ 12,61]) et d'élévation de la CRP.

\section{Hematologic, biochemical and immune biomarker abnormalities associated with severe illness and mortality in coronavirus disease 2019 (Covid-19): a meta-analysis}

Henry BM, De Oliveira MHS, Benoit S, et al (2020) Clin Chem Lab Med [in press]

Méta-analyse incluant 21 études dont l'objectif est d'identifier les marqueurs biologiques associés à la sévérité des patients atteints de Covid-19. Les patients sévères par rapport aux non-sévères présentent une diminution de l'hémoglobine et une augmentation des leucocytes. Les patients décédés par rapport aux survivants ont une augmentation des leucocytes, de la bilirubine totale, de la créatine phosphokinase, de la ferritine et de l'interleukine-6. Ils présentent également une diminution des lymphocytes et des plaquettes. 
Acute heart failure in multisystem inflammatory syndrome in children (MIS-C) in the context of global SARS-CoV-2 pandemic

Belhadjer Z, Méot M, Bajolle F, et al (2020) Circulation [in press]

Étude française multicentrique, observationnelle, incluant 35 enfants admis en réanimation pour choc cardiogénique, dysfonction ventriculaire gauche et état inflammatoire sévère. Le diagnostic d'infection par le SARS-CoV-2 était retrouvé par réaction en chaîne par polymérase (PCR) chez $88 \%$ des enfants. Dix sur 35 ont été placés temporairement en assistance circulatoire. Ils ont tous été traités par des immunoglobulines associées parfois à des corticö̈des. Aucun décès n'était à déplorer dans cette série.

\section{A trial of lopinavir-ritonavir in adults hospitalized with severe Covid-19}

Cao B, Wang Y, Wen D, et al (2020) N Engl J Med 382:1787-99

Étude chinoise monocentrique, randomisée, interventionnelle, incluant 199 adultes présentant une infection sévère à Covid-19. Les auteurs comparent le temps nécessaire à une amélioration clinique entre les patients traités par l'association lopinavir $400 \mathrm{mg} \times 2 / \mathrm{j}$ et ritonavir $100 \mathrm{mg} \times 2 / j$ vs des soins classiques. Les auteurs ne retrouvent pas de différence ni sur l'amélioration clinique (hasard ratio $=1,24$; intervalle de confiance à $95 \%$ : [0,90-1,72]), ni sur la mortalité à $28 j$ (19,2 vs $25 \%$ ), ni sur la charge virale.

\section{Clinical efficacy of hydroxychloroquine in patients} with Covid-19 pneumonia who require oxygen: observational comparative study using routine care data

Mahévas M, Tran V, Roumier M, et al (2020) Bmj 369:m1844

Étude française multicentrique, observationnelle, incluant 181 adultes présentant une infection à Covid-19. Les auteurs comparent le devenir entre les patients traités par hydroxychloroquine (HCQ ; $600 \mathrm{mg}$ par $\mathrm{j} ; \mathrm{n}=84$ ) et les autres. Le taux de survie, sans transfert en soins intensifs à $21 j$, était de $76 \%$ dans le groupe HCQ vs $75 \%$ dans le groupe témoin (différence non significative). La survie globale à 21 jétait de $89 \%$ dans le groupe $H C Q$ vs $91 \%$ dans le groupe témoin (ns), et la survie sans SDRA était de $69 \%$ dans le groupe HCQ vs $74 \%$ dans le groupe témoin (ns).
Hydroxychloroquine or chloroquine with or without a macrolide for treatment of Covid-19: a multinational registry analysis

Mehra M, Desai S, Ruschitzka F, Patel A (2020) Lancet [in press]

Étude internationale, multicentrique, observationnelle, incluant 96032 patients hospitalisés pour une infection à Covid-19 (mortalité intrahospitalière $=11 \%$ ). Les auteurs comparent la mortalité intrahospitalière entre les patients traités par hydroxychloroquine (HCQ) ou chloroquine (CQ), avec ou sans macrolides, et ceux traités sans HCQ. Après ajustement sur les facteurs confondants et comparés au groupe témoin, les trois groupes étudiés (HCQ, $H C Q+$ macrolides, $C Q+$ macrolides) étaient associés à une surmortalité (hasard ratios et intervalles de confiance à $95 \%$, respectivement, de $1,33[1,22-1,45] ; 1,44[1,36-$ $1,53] ; 1,36[1,27-1,46])$.

Remdesivir in adults with severe Covid-19: a randomised, double-blind, placebo-controlled, multicentre trial

Wang Y, Zhang D, Du G, et al (2020) Lancet 395:1569-78

Étude chinoise multicentrique, randomisée, interventionnelle, incluant 237 adultes hospitalisés pour une infection à Covid-19 (mortalité à $28 j=13 \%$ ). Les auteurs comparent le temps nécessaire à une amélioration clinique entre les patients traités par remdesivir (200 mg puis $100 \mathrm{mg} / \mathrm{j}$ durant $10 j$ ) vs placebo. Le temps d'amélioration médian dans le bras remdesivir n'est pas différent du bras placebo, 21 vs $23 j$ respectivement (hasard ratio $=1,23$; intervalles de confiance à $95 \%$ : [0,87-1,75]). Les auteurs ne retrouvent pas de différence en termes de mortalité.

\section{Remdesivir for the treatment of Covid-19, preliminary Report}

Beigel JH, Tomashek KM, Dodd LE, et al (2020) N Engl J Med [in press]

Étude américaine multicentrique, randomisée, interventionnelle, incluant 1059 adultes hospitalisés pour une infection à Covid-19 (mortalité à $14 j=8,1 \%$ ). Les auteurs comparent le temps nécessaire à une amélioration clinique entre les patients traités par remdesivir $(200 \mathrm{mg}$ puis $100 \mathrm{mg} / \mathrm{j}$ durant $9 j$ ) vs placebo. Les patients traités par remdesivir ont eu un temps d'amélioration médian de $11 j$ (intervalles de confiance à $95 \%$ : [9-12]), vs 15 j [13-19] dans le groupe placebo (hasard ratio $=1,32$ [1,12-1,55]). Les estimations de Kaplan-Meier concernant la mortalité à $14 j$ étaient de 7,1\% avec le remdesivir vs $12 \%$ avec le placebo $(H R=0,70[0,47-1,04])$. 


\section{Références}

1. Huang C, Wang Y, Li X, et al (2020) Clinical features of patients infected with 2019 novel coronavirus in Wuhan, China. Lancet 395:497-506

2. Wang D, Hu B, Hu C, et al (2020) Clinical characteristics of 138 hospitalized patients with 2019 novel coronavirus-infected pneumonia in Wuhan, China. JAMA [in press]

3. Chen N, Zhou M, Dong X, et al (2020) Epidemiological and clinical characteristics of 99 cases of 2019 novel coronavirus pneumonia in Wuhan, China: a descriptive study. Lancet 395:507-13

4. Chen L, Liu HG, Liu W, et al (2020) Analysis of clinical features of 29 patients with 2019 novel coronavirus pneumonia. Zhonghua Jie He He Hu Xi Za Zhi 43:E005

5. Novel coronavirus pneumonia emergency response epidemiology team (2020) The epidemiological characteristics of an outbreak of
2019 novel coronavirus diseases (Covid-19) in China. Zhonghua Liu Xing Bing Xue Za Zhi 41:145-51

6. Xu X, Wu X, Jiang X, et al (2020) Clinical findings in a group of patients infected with the 2019 novel coronavirus (SARS-Cov-2) outside of Wuhan, China: retrospective case series. Bmj 368:m606

7. Huang C, Wang Y, Li X, et al (2020) Clinical features of patients infected with 2019 novel coronavirus in Wuhan, China. Lancet 395:497-506

8. Zhou F, Yu T, Du R, et al (2020) Clinical course and risk factors for mortality of adult inpatients with Covid-19 in Wuhan, China. Lancet [in press]

9. Zou L, Ruan F, Huang M, et al (2020 SARS-CoV-2 viral load in upper respiratory specimens of infected patients. N Engl J Med [in press]

10. Pan F, Ye T, Sun P, et al (2020) Time course of lung changes on chest CT during recovery from 2019 novel coronavirus (Covid-19) pneumonia. Radiology [in press] 